\title{
Fundus changes in persistent and recurrent choroidal folds
}

\author{
FRANK W. NEWELL \\ From the Eye Research Laboratories, the University of Chicago, the Pritzker School of Medicine, \\ 939 East 57th Street, Chicago, Illinois 60637, USA.
}

SUMMARY Linear bead-like pigmentation in choroidal folds and diffuse nonspecific retinal pigment epithelial proliferation are seen in long-study choroidal folds. In addition there may be linear streaks of scleral staining.

Until the advent of fluorescein angiography, horizontal and vertical folds, grooves and striae of the choroid, retinal pigment epithelium, and sensory retina were considered to be unusual changes secondary to orbital tumours. In the 1968 Lang lecture Norton ${ }^{1}$ described their fluorescent pattern, and in a subsequent publication he and $\mathrm{Kroll}^{2}$ described their regression. In only 4 of the 10 cases described initially by Norton were choroidal folds associated with tumours; it was evident that those folds occurred in many conditions other than orbital tumour. Newell, ${ }^{3}$ in the 1972 Gradle lecture, described 16 patients with choroidal folds that were associated with a variety of ocular disorders.

Recently we examined patients who had either persistent or recurrent choroidal folds. Pigment proliferation and attenuation of the retinal pigment epithelium with linear staining of the sclera provided a characteristic picture not previously emphasised.

\section{Case reports}

CASE 1

A 47-year-old man was first seen with a haemangioma in the muscle cone of the right orbit. Four years after excision, although other signs of tumour had disappeared, the choroidal folds persisted. The peaks of some of the folds were discretely pigmented. Ten years later the tumour recurred, with choroidal folds and pigment. After excision, the folds disappeared, leaving a residue of pigment proliferation that involved both the nasal and temporal portions of the fundus. In several areas the pigment occurred in isolated dots; in others a series of discrete, linear,

Correspondence to Frank W. Newell, MD. bead-like dots were observed (Fig. 1). There were broad discrete bands of hypopigmentation corresponding to earlier folds.

Fluorescein angiography (Fig. 2) demonstrated far more severe fundus changes than evident with light ophthalmoscopy. There were conspicuous broad bands of hyperfluorescence in the late fluorescein phases. The central portion of these bands contained pigment arranged continuously in a linear pattern. In some areas the pigment was discrete. There was far

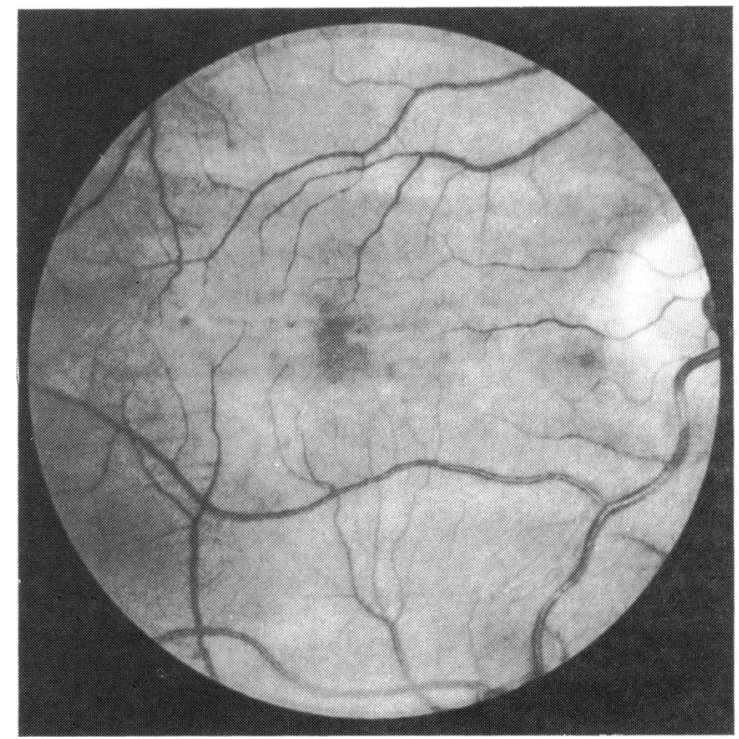

Fig. 1 Case 1. Right posterior eyegrounds. Discrete, whitish, linear streaks mark location of previous choroidal folds. There are discrete dots of pigment, some temporal to the fovea centralis and arranged in a linear pattern. 


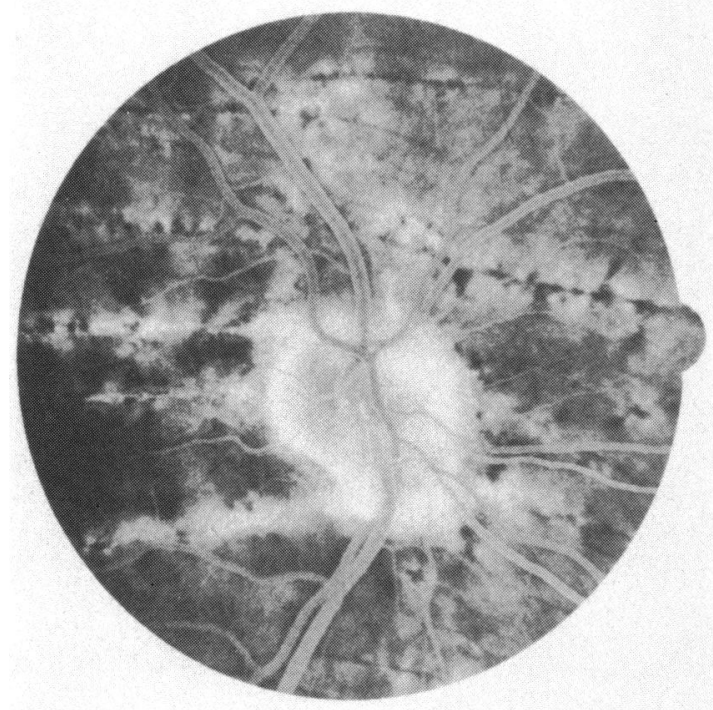

Fig. 2 Case 1. Late venous phase of fluorescein angiogram. There are areas of hyperfluorescence with striking aggregates of pigment.

more pigment than was visible either ophthalmoscopically or with Kodachrome photographs of the fundus. There was no fluorescein leakage. In late fluorescein (Fig. 3) there was fluorescence of the disc and linear striae of fluorescence that involved both the nasal and temporal side of the disc. This hyper-

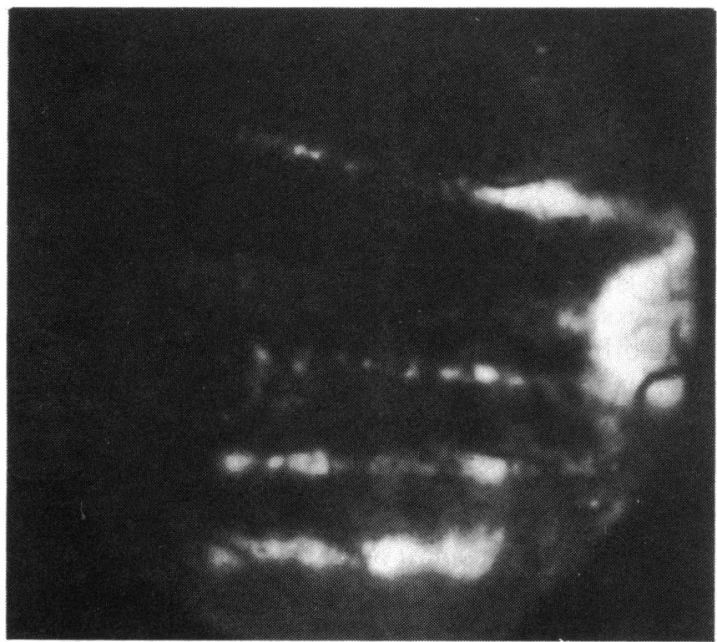

Fig. 3 Case 1. Fluorescein angiogram 8 minutes after injection. There are broad, irregular, linear streaks of hyperfluorescence with fluorescein transmission interrupted by aggregates of pigment. There is no fluorescein leakage.

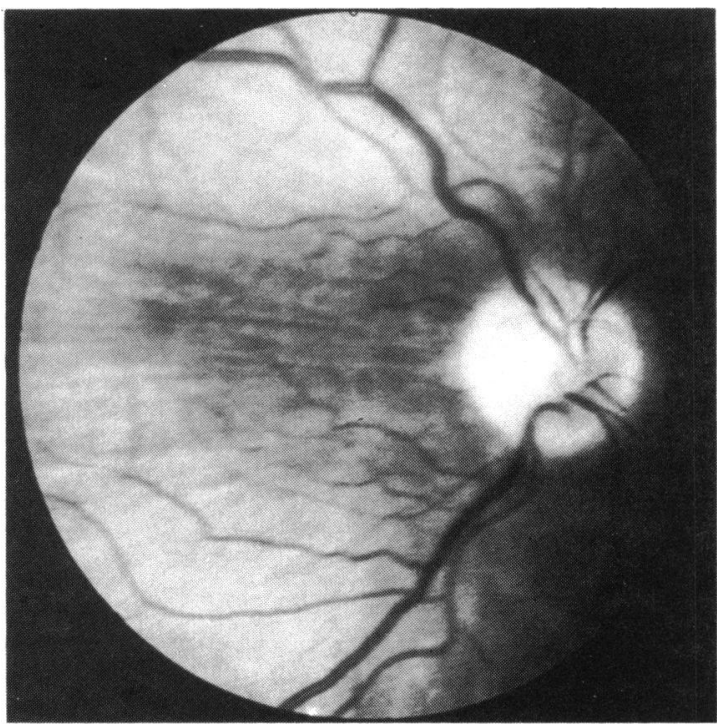

Fig. 4 Case 2. Right posterior eyegrounds. Ill-defined whitish linear streaks are present.

fluorescence arose with scleral staining. There was no fluorescein leakage.

\section{CASE 2}

A 37-year-old woman was first seen because of a right papilloedema. Choroidal folds were present above and below the disc; the cerebrospinal fluid pressure was elevated; extensive neurological and radiological examinations were negative. Pseudotumor cerebri was diagnosed, and she was treated with prednisone. Four years later, after intermittent prednisone therapy, the folds were slightly pigmented; the nasal margins of the optic disc were blurred. Thirteen years later vision was normal in each eye with hypermetropic correction. The disc margins were indistinct, but the disc was flat. Choroidal folds persisted as parallel, broad, whitish lines, above and below the disc. On fluorescein angiography choroidal folds that were far more coarse and granular than those of 10 years earlier were seen (Fig. 4). In addition there were persistent linear areas that did not increase in size near the disc (Figs. 5, 6).

CASE 3

A 50-year-old man had a left proptosis of one year's duration that was subsequently corrected with removal of a left frontal sinus mucocele. The retinal blood vessels were excessively tortuous, and parallel choroidal folds were present in the temporal posterior fundus. There were diffuse areas of pigmentation. Fluorescein angiography indicated a broad streak of hyperfluorescence adjacent to the disc (Fig. 7) that was evident in the arterial phase. It was not present in 


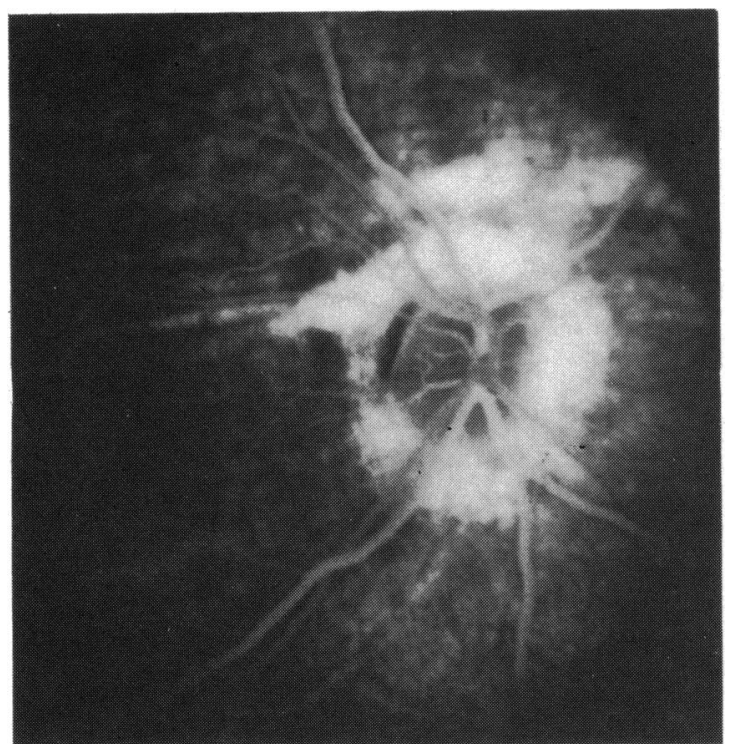

Fig. 5 Case 2. Late venous phase of fluorescein angiogram showing coarse choroidal background with ill-defined linear streaks.

the late venous phase. The initial appearance could be confused with a choriocapillaris dye leakage.

\section{CASE 4}

A 29-year-old man had a right proptosis since birth with several unsuccessful surgical attempts at correction. There were temporal choroidal folds (Fig. 8). The inferior temporal fundus demonstrated diffuse patches of pigment aggregation. These pigmented areas were irregular in shape, of varying size, and not related directly to visible choroidal folds. The cornea was abraded and treatment was directed toward corneal exposure, and he did not return.

\section{Discussion}

Ophthalmoscopically in their early stages choroidal folds appear as alternating bright and dark lines involving predominantly the posterior pole. The peaks of the pole are bright and the valleys are a darker shade of red. The folds are approximately parallel and tend to vary in both length and width. They do not extend beyond the equator. They occur more commonly on the temporal side of the disc than on the nasal side. Horizontal folds may pass above and below the disc. There may be vertical folds that lie on either the temporal or medial side of the disc or above or below it. Occasionally the folds are slanted. They are never precisely horizontal or vertical.

The choroidal folds are best observed by direct illumination using the direct ophthalmoscope or bio-

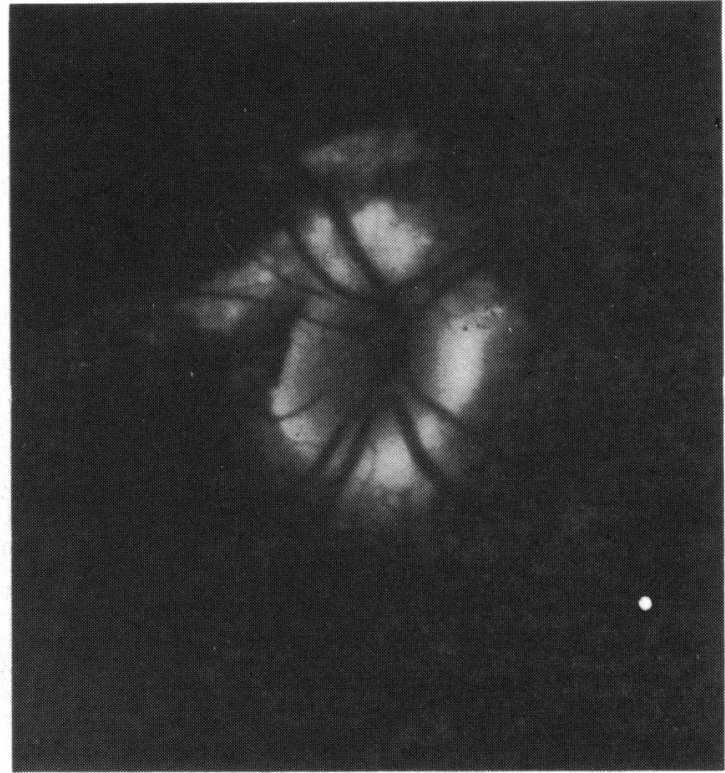

Fig. 6 Case 2. Fluorescein angiogram 10 minutes after injection showing broad streak of scleral staining above disc.

microscopy combined with a fundus lens. With direct illumination the light is directed to the area adjacent to the lesion to be studied and attention directed to the junction of the edge of the light and the lesion. The alternating bright and dark lines are evident. The folds vary in number from 2 or 3 to 20 or more. In the early stages they may be narrow as small blood vessels but gradually become wider with smooth edges.

After fluorescein injection the peaks fluoresce during the filling of the choriocapillaris, and the valleys remain dark. The contrast between peaks and valleys becomes more marked during the subsequent arterial and venous phases and creates a striking picture of alternating bright and dark lines. Often many more folds are demonstrated with fluorescein angiography than are seen with light ophthalmoscopy. There is no leakage of fluorescein.

Choroidal folds are seen in association with spacefilling orbital lesions such as tumours or thyroid ophthalmopathy, in papilloedema, ocular hypotony, scleral shrinkage due to either scleritis or scleral buckling, and severe hypermetropia. They may also be seen in choroidal tumours, subretinal neovascularisation, uveitis, and familially. Sometimes no cause is found. Folds have not been described in eyes with myopia.

The main condition to be differentiated is retinal folds, which, however, may occur spontaneously. Retinal folds do not cause an abnormal fluorescein pattern, are usually associated with vitreous humour abnormalities, and may show increased tortuosity of 


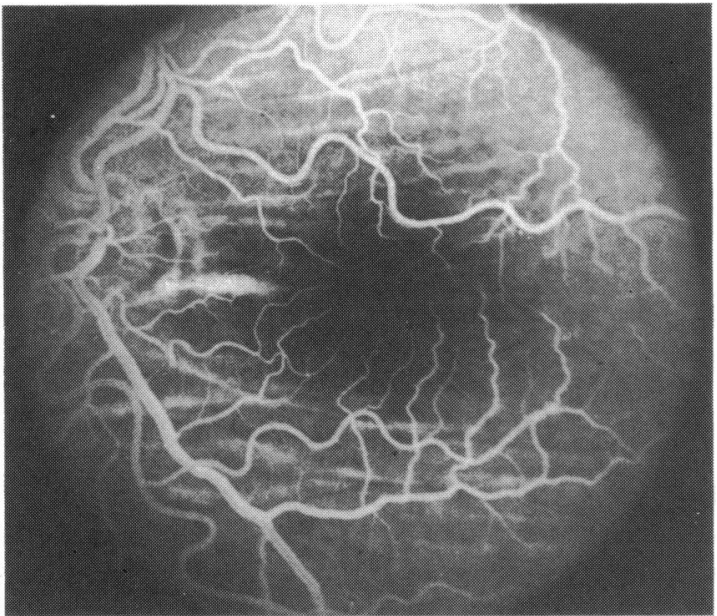

Fig. 7 Case 3. Late venous fluorescein angiogram showing a linear streak of hyperfluorescence which did not persist in the later venous phases.

retinal blood vessels. Ophthalmoscopy with retroillumination readily distinguishes choroidal folds.

Nettleship ${ }^{4}$ first described choroidal folds in 1884 . The patient, a 36-year-old man, had syphilis at age 30 , and thereafter developed a brain tumour that extended to the right orbit. A secondary optic atrophy was present. The illustration, in addition to the optic atrophy, shows choroidal folds both temporal and nasal to the optic disc. The folds in the temporal region are pigmented with discrete, closely packed pigment particles arranged in a linear pattern. The pigment is mainly located at the upper portion of the fold. The nasal choroidal folds are less conspicuously pigmented but again in a linear pattern. Below the optic disc there is diffuse pigmentation not associated with folds. In 1954 Dellaporta $^{5}$ described bilateral choroidal folds secondary to operative hypotony that caused papilloedema. Discrete pigment nasal to the disc without a linear pattern occurred in several areas. Less conspicuous diffuse pigmentation was present in the fellow eye.

These 2 reports indicate the major patterns of pigmentation occurring in choroidal folds. The most characteristic consists of small pigmented dots, almost bead-like, that extend along a choroidal fold. The pigmentation appears on the slope of the fold rather than in the peak or the valley. Immediately adjacent to the pigmentation is a discrete, minute area of depigmentation. The stellate shaped area of pigmentation and the linear streaks of depigmentation seen in case 1 on fluorescein angiography are unique changes. They appear to occur because of prolonged choroidal folds. A more diffuse type of

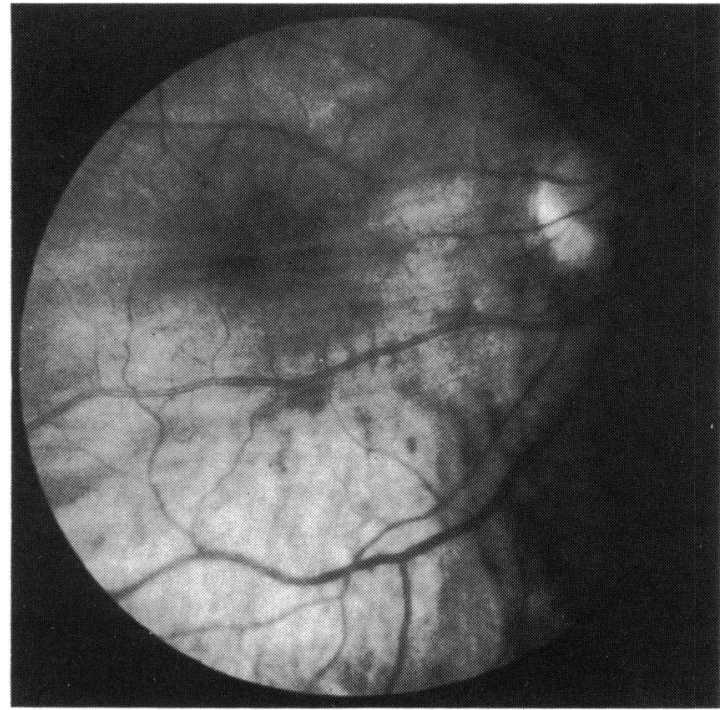

Fig. 8 Case 4. Diffuse pigment of right posterior pole with choroidal folds passing through fovea.

pigmentation, together with a persistent pattern of choroidal folds, was evident in case 2. Far more marked changes were evident with fluorescein angiography than with light ophthalmoscopy and retroillumination. Diffuse, patchy, nonspecific pigmentation seen in case 4 is similar to that described by Dellaporta.

Hyperfluorescent streaks are seen in 2 different situations. Friberg and Grove ${ }^{6}$ directed attention to the broad streaks of hyperfluorescence as seen in case 3 . This hyperfluorescence does not persist and is related to whatever mechanism is responsible for choroidal folds. The hyperfluorescence seen in the late fluorescein phases in cases 1 and 2 appears to arise with scleral staining, evident through areas of attenuation of pigment in the retinal pigment epithelial layer.

This study was supported in part by a grant from Research to Prevent Blindness, Inc.

\section{References}

1 Norton EWD. A characteristic fluorescein angiographic pattern in choroidal folds. Proc R Soc Med 1969; 62: 119-28.

2 Kroll AJ, Norton EWD. Regression of choroidal folds. Trans Am Acad Ophthalmol Otolaryngol 1970; 74: 515-25.

3 Newell FW. Choroidal folds. The seventh Harry Searls Gradle Memorial Lecture. Am J Ophthalmol 1973; 75: 903-42.

4 Nettleship E. Peculiar lines in the choroid in a case of postpapillitic atrophy. Trans Ophthalmol Soc UK 1884; 4: 167-8.

5 Dellaporta A. Fundus changes in hypotony. Am J Ophthalmol 1955; 40: 781-5.

6 Friberg TR, Grove AS. Choroidal folds and refractive errors associated with orbital tumors. Arch Ophthalmol 1983; 101: 598-603. 